\title{
Synthesis and characterization of core/shell $\mathrm{Fe}_{3} \mathrm{O}_{4} / \mathrm{ZnSe}$ fluorescent magnetic nanoparticles
}

\author{
José M. Vargas, ${ }^{1}$ Amber A. McBride, ${ }^{1}$ John B. Plumley, ${ }^{1}$ Yann Fichou, ${ }^{1, a)}$ Tosifa A. Memon, ${ }^{1}$ \\ Vichiksha Shah, ${ }^{1}$ Nathaniel C. Cook, ${ }^{1}$ Brian A. Akins, ${ }^{1}$ Antonio C. Rivera, ${ }^{1}$ \\ Gennady A. Smolyakov, ${ }^{1}$ James R. O'Brien, ${ }^{2}$ Natalie L. Adolphi, ${ }^{3}$ Hugh D. C. Smyth, ${ }^{4}$ \\ and Marek Osiński ${ }^{1, b)}$ \\ ${ }^{1}$ Center for High Technology Materials, University of New Mexico, Albuquerque, New Mexico 87106-4343, \\ USA \\ ${ }^{2}$ Quantum Design, San Diego, California 92121, USA \\ ${ }^{3}$ Health Sciences Center, University of New Mexico, Albuquerque, New Mexico 87131, USA \\ ${ }^{4}$ College of Pharmacy, University of Texas at Austin, Austin, Texas 78712, USA
}

(Presented 17 November 2010; received 25 September 2010; accepted 22 December 2010; published online 8 April 2011)

\begin{abstract}
We report on the successful preparation and characterization of fluorescent magnetic core/shell $\mathrm{Fe}_{3} \mathrm{O}_{4} / \mathrm{ZnSe}$ nanoparticles (NPs) with a spherical shape by organometallic synthesis. The $7 \mathrm{~nm}$ core $/ 3 \mathrm{~nm}$ shell NPs show good magnetic and photoluminescence (PL) responses. The observed PL emission/excitation spectra are shifted to shorter wavelengths, compared to a reference $\mathrm{ZnSe} \mathrm{NP}$ sample. A dramatic reduction of PL quantum yield is also observed. The temperature dependence of the magnetization for the core/shell NPs shows the characteristic features of two coexisting and interacting magnetic $\left(\mathrm{Fe}_{3} \mathrm{O}_{4}\right)$ and nonmagnetic $(\mathrm{ZnSe})$ phases. Compared to a reference $\mathrm{Fe}_{3} \mathrm{O}_{4} \mathrm{NP}$ sample, the room-temperature Néel relaxation time in core/shell NPs is three times longer.

(C) 2011 American Institute of Physics. [doi:10.1063/1.3565190]
\end{abstract}

Engineered multifunctional nanoparticles (NPs) with highly integrated imaging modalities are a key focus area in bionanotechnology that will have a profound impact on molecular diagnosis, imaging, and therapeutics. ${ }^{1-3}$ However, combining multiple components on a nanometer scale to create new imaging modalities not available from the individual components has proven to be challenging. ${ }^{4}$ An example of such multifunctional NPs are fluorescent magnetic NPs that bear two attractive features, fluorescence and superparamagnetism, allowing their intracellular movements to be controlled using magnetic force and monitored using a fluorescent microscope. These features could lead to effective multifunctional drug-loaded magnetic NPs that would facilitate increased drug transport rates, mucus penetration, and antibiotic efficiency in biofilms. ${ }^{1}$ In this paper, we have used a two-step solution phase route to prepare cadmium-free core/ shell $\mathrm{Fe}_{3} \mathrm{O}_{4} / \mathrm{ZnSe}$ colloidal NPs with a spherical shape. Both individual components show good characteristics in terms of good chemical stability, biocompatibility, and low toxicity.

Bulk magnetite $\mathrm{Fe}_{3} \mathrm{O}_{4}$ is a mixed-valence $3 d$ transition metal compound. It has an inverted cubic spinel structure, in which tetrahedral $A$ sites contain one-third of the $\mathrm{Fe}$ ions as $\mathrm{Fe}^{3+}$, whereas octahedral $B$ sites contain the remaining $\mathrm{Fe}$ ions, with equal numbers of $\mathrm{Fe}^{2+}$ and $\mathrm{Fe}^{3+}$ at $B 1$ and $B 2$ sites, respectively. Below $860 \mathrm{~K}$, bulk magnetite is ferromagnetic, with the $A$-site magnetic moments aligned antiparallel to the $B$-site moments. Photoemission measurements clearly showed a gap of $\sim 0.14 \mathrm{eV}$ in the spectra. ${ }^{5}$

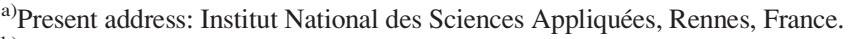

${ }^{b)}$ Author to whom correspondence should be addressed. Electronic mail: osinski@chtm.unm.edu.
}

$\mathrm{ZnSe}$ has a room temperature bulk bandgap of $2.7 \mathrm{eV}$ $(460 \mathrm{~nm})$. ZnSe shells have long been used as a capping material for CdSe-core quantum dots (QDs) for surface passivation and biological labeling reagents. The organometallic synthesis of pure band-edge fluorescent $\mathrm{ZnSe}$ size-tunable QDs has been reported to result in highly luminescent UVblue NP materials with bandgaps tunable between 2.8 and 3.4 $\mathrm{eV}$ and a quantum yield (QY) between $20 \%$ and $50 \% .{ }^{6}$ More recently, Cozzoli et al. studied the shape and phase control of colloidal ZnSe NPs, where the as-prepared NPs exhibited distinguishable, shape-dependent optical properties in the UVblue region. ${ }^{7}$ They observed that a red-shift of the bandgap in longer nanorods, or in general branched nanostructures with long rod sections, was generally accompanied by a significant decrease in the photoluminescence (PL) QY, which could be much lower than $1 \%$ for the branched structures.

Considering the characteristics of both individual materials described above, we note that in the simple approach of dimer particles, i.e., magnetite NPs attached to semiconductor QDs, such as ZnSe, PL emission from these multifunctional NPs should be expected to be strongly reduced due to the fact that magnetite is a semimetal with a much smaller bandgap than $\mathrm{ZnSe}$, providing a leakage path to electrons excited in ZnSe. Similar problem should be expected with multifunctional NPs involving other semiconductor materials, such as CdSe with a bulk bandgap of $\sim 1.75 \mathrm{eV}$. However, it has been shown that multicomponent $\mathrm{CdSe} /$ iron oxide core/shell and dimer NPs can have good PL efficiency. ${ }^{4}$

All NPs described in this paper were synthesized using standard air-free procedures and commercially available reagents. The main reaction involves the nucleation and growth of nominally $\mathrm{Fe}_{3} \mathrm{O}_{4}$ NPs with a mean size of $7 \mathrm{~nm}$ 


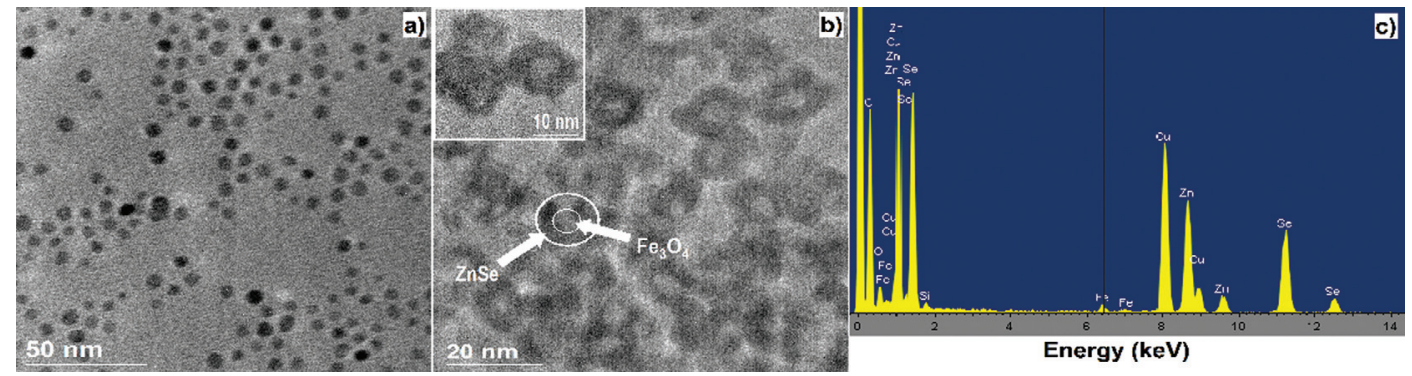

FIG. 1. (Color online) TEM image of (a) reference $\mathrm{Fe}_{3} \mathrm{O}_{4}$ NPs and (b) core/shell $\mathrm{Fe}_{3} \mathrm{O}_{4} / \mathrm{ZnSe} \mathrm{NPs}$. The white circles indicate the $\mathrm{Fe}_{3} \mathrm{O}_{4}$ core (lighter) and ZnSe shell (darker). Inset: HR-TEM image of the core/shell $\mathrm{Fe}_{3} \mathrm{O}_{4} / \mathrm{ZnSe} \mathrm{NPs}$. (c) EDS spectrum measured for the core/shell NPs. The Cu and $\mathrm{C}$ peaks originate from the holding grid.

and a size deviation of $\sim 1.2 \mathrm{~nm}$. Briefly, following a modified procedure of Sun and Zeng, ${ }^{8}$ a solution of iron (III) acetylacetonate $\left[\mathrm{Fe}(\mathrm{acac})_{3}, 2 \mathrm{mmol}\right]$ in the presence of 1,2 hexadecanediol $(10 \mathrm{mmol})$ and the surfactants oleic acid (6 mmol) and oleylamine (OLA, $6 \mathrm{mmol})$ in octyl ether $(30 \mathrm{~mL})$ was heated to reflux for $1 \mathrm{~h}$. The obtained blackbrown reference sample was labeled as $\mathrm{Fe}_{3} \mathrm{O}_{4}$ NPs and was used thereafter as a seed for the core/shell $\mathrm{Fe}_{3} \mathrm{O}_{4} / \mathrm{ZnSe} \mathrm{NP}$ growth. In a typical procedure, modified after Zhong et al., ${ }^{9}$ $2 \mathrm{~mL}$ of the reference $\mathrm{Fe}_{3} \mathrm{O}_{4} \mathrm{NP}$ sample in 1-octadecene (ODE, $0.05 \mathrm{~g} / \mathrm{mL}$ ) was transferred into a reaction flask and mixed with $8.4 \mathrm{mmol}$ of OLA and ODE $(3 \mathrm{~mL})$ at $120^{\circ} \mathrm{C}$ in an inert $\mathrm{Ar}$ atmosphere under vigorous stirring. Subsequently, zinc oleate/OLA/ODE precursor $(6.3 \mathrm{~mL})$ was injected into the flask at $120^{\circ} \mathrm{C}$, and the solution was purged with $\mathrm{Ar}$ for $30 \mathrm{~min}$. The solution was then heated up to $300^{\circ} \mathrm{C}$, and a selenium powder/trioctyl phosphine/ODE mixture $(6 \mathrm{~mL})$ was quickly injected. After $10 \mathrm{~min}$ at $300{ }^{\circ} \mathrm{C}$, the final black-brown solution was cooled to room temperature. The obtained NPs were easily dispersed in nonpolar solvents such as hexane or chloroform. This sample was labeled as $\mathrm{Fe}_{3} \mathrm{O}_{4} / \mathrm{ZnSe}$ NPs. Alternatively, the light-yellow reference sample of ZnSe NPs with a mean size of $3.5 \mathrm{~nm}$ and a narrow size distribution $(\sigma=0.15)$ was synthesized following the modified procedure of Zhong et al. ${ }^{9}$ This reference sample was labeled as ZnSe NPs.

Figure 1 shows TEM images of $\mathrm{Fe}_{3} \mathrm{O}_{4}$ NPs [Fig. 1(a)] and core/shell $\mathrm{Fe}_{3} \mathrm{O}_{4} / \mathrm{ZnSe}$ NPs [Fig. 1(b)]. For the core/shell NPs [Fig. 1(b)], the TEM images confirm the presence of regions of two different contrasts in the morphology of the majority of the particles. Roughly, the TEM signal at every projected point of a particle with a constant density is inversely proportional to $Z^{2}$, with the thickness of the material being much less important. ${ }^{10}$ Therefore, the ZnSe particles, which have a higher $Z$ (mean value of 32) compared with the $\mathrm{Fe}_{3} \mathrm{O}_{4}$ particles (mean value of $Z \approx 15.7$ ), are imaged as darker objects. From the shape of the particles, we can infer that $\mathrm{Fe}_{3} \mathrm{O}_{4}$ NPs play the role of seeding sites for $\mathrm{ZnSe}$ growth, leading to the appearance of core/shell spherical NPs. The size of the core/shell NPs is estimated as a diameter of $6.8 \pm 1.5 \mathrm{~nm}$ for $\mathrm{Fe}_{3} \mathrm{O}_{4}$ cores and a thickness of $3.8 \pm 1.2 \mathrm{~nm}$ for $\mathrm{ZnSe}$ shells, which is in a good agreement with the diameter of $7 \mathrm{~nm}$ for the reference $\mathrm{Fe}_{3} \mathrm{O}_{4}$ sample. The elemental presence of $\mathrm{Zn}, \mathrm{Se}$, and $\mathrm{Fe}$ in the composition of the synthesized core/shell NPs was verified by energy dispersive $\mathrm{x}$-ray spectroscopy (EDS), as shown in Fig. 1(c).
Figure 2 shows the XRD patterns $\left(\mathrm{CuK}_{\alpha}, \lambda_{\text {ave }}=0.15418 \mathrm{~nm}\right)$ for the $\mathrm{ZnSe}$ and $\mathrm{Fe}_{3} \mathrm{O}_{4}$ reference samples and for the core/shell $\mathrm{Fe}_{3} \mathrm{O}_{4} / \mathrm{ZnSe}$ sample. The pattern for the $\mathrm{ZnSe} \mathrm{NPs}$ [Fig. 2(a)] indicates that the NPs have a noncentrosymmetric cubic $F 43 m$ symmetry, as expected from the bulk crystal structure. The peaks are broader for the smaller nanocrystals, but the pattern is centered on the bulk crystal lines, with no other features. For the $\mathrm{Fe}_{3} \mathrm{O}_{4}$ NPs [Fig. 2(b)], the broad and relatively low signal-to-noise ratio peaks corresponding to magnetite $\mathrm{Fe}_{3} \mathrm{O}_{4}$ (cubic phase, $F d 3 m$ ) suggest a short crystalline order and an internal polycrystalline structure in the particles. Taking the full width at half-maximum of the most intense peak and using the Scherrer equation, we determined the lower bound on the average crystalline size to be $4.0 \pm 0.9 \mathrm{~nm}$ for the $\mathrm{ZnSe} \mathrm{NPs}$ and $4.7 \pm 1.5 \mathrm{~nm}$ for the $\mathrm{Fe}_{3} \mathrm{O}_{4}$ NPs. While these values are in good agreement with the morphological information obtained from the TEM images for the

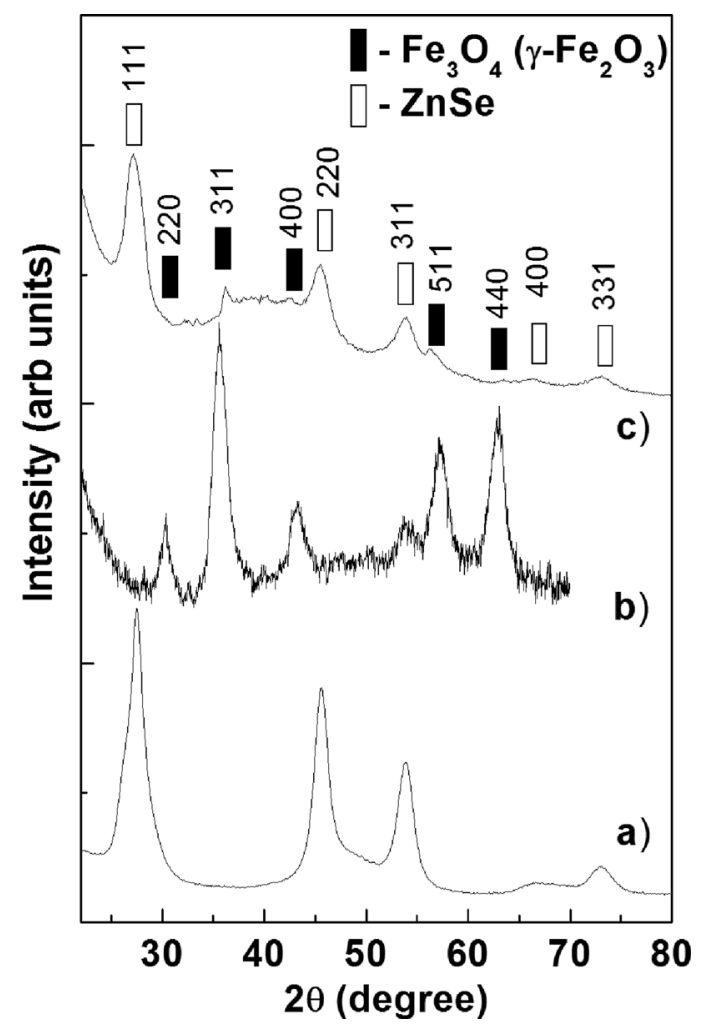

FIG. 2. Powder XRD patterns for the reference (a) ZnSe NPs, (b) $\mathrm{Fe}_{3} \mathrm{O}_{4}$ NPs, and (c) core/shell $\mathrm{Fe}_{3} \mathrm{O}_{4} / \mathrm{ZnSe}$ NPs. The positions of the $\mathrm{ZnSe}$ and $\mathrm{Fe}_{3} \mathrm{O}_{4}$ (coinciding with $\mathrm{Fe}_{2} \mathrm{O}_{3}$ ) lines taken from the International Centre for Diffraction Data database are indicated at the top of the figure. 

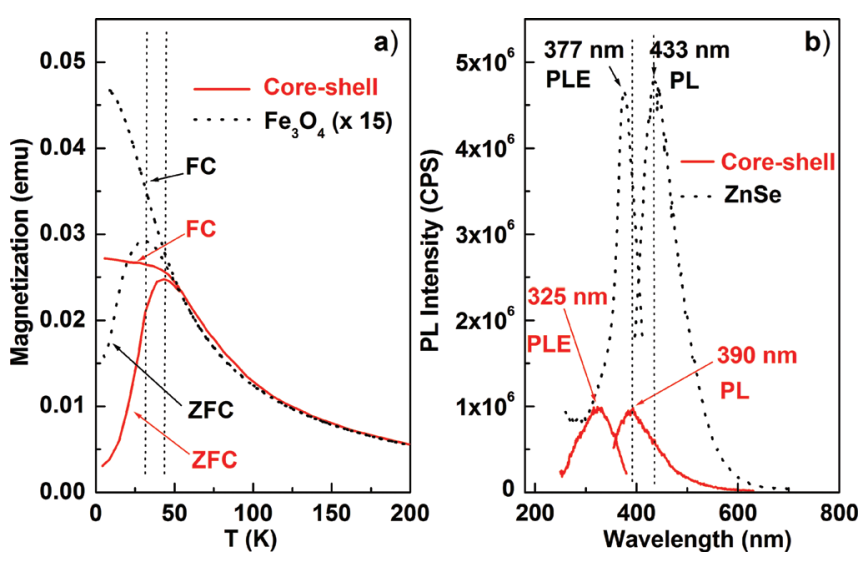

FIG. 3. (Color online) (a) Magnetization against $T$ under ZFC and FC conditions for the reference $\mathrm{Fe}_{3} \mathrm{O}_{4}$ and core/shell $\mathrm{Fe}_{3} \mathrm{O}_{4} / \mathrm{ZnSe}$ NP samples. (b) PLE and PL spectra measured at room temperature for the reference $\mathrm{ZnSe}$ and core/shell $\mathrm{Fe}_{3} \mathrm{O}_{4} / \mathrm{ZnSe} \mathrm{NP}$ samples.

ZnSe NPs, the agreement is not so good for the $\mathrm{Fe}_{3} \mathrm{O}_{4}$ NPs. The discrepancy in particle sizes obtained from TEM and XRD analyses has been frequently observed in this type of iron oxide compound synthesized by chemical methods, which is explained by polycrystalline structure of the compound.

It should be noted that the lattice parameters of the spinel structures, magnetite $\left(\mathrm{Fe}_{3} \mathrm{O}_{4}\right)$ and maghemite $\left(\gamma-\mathrm{Fe}_{2} \mathrm{O}_{3}\right)$, are quite similar. Because the XRD lines of the NP samples are broadened, it is difficult to distinguish between magnetite and maghemite NPs by XRD. ${ }^{8,11}$ However, the decomposition of $\mathrm{Fe}(\mathrm{acac})_{3}$ at high temperature has been claimed to lead to magnetite as the final product ${ }^{8}$; hence we ascribe a magnetite structure to our iron oxide NPs.

The $\mathrm{Fe}_{3} \mathrm{O}_{4} / \mathrm{ZnSe}$ XRD pattern [Fig. 2(c)] shows a strong contribution from the peaks originating from $\mathrm{ZnSe}$ and a much weaker contribution from the broad peaks of magnetite. The detailed analysis of the peak positions and their relative intensities confirms a lattice expansion for the magnetite that has been estimated to be $d_{511}=1.64(3) \AA$, which is $2 \%$ larger than the corresponding value for the reference $\mathrm{Fe}_{3} \mathrm{O}_{4}$ sample $\left[\mathrm{d}_{511}=1.60(5) \AA\right]$, a tendency also observed in dimer $\mathrm{Ag}-\mathrm{Fe}_{3} \mathrm{O}_{4}$ NPs. ${ }^{10}$ At the same time, no sign of lattice distortion is observed in the ZnSe-related peaks in the core/shell NPs.

Figure 3(a) shows the $T$ dependence of the magnetization for the reference $\mathrm{Fe}_{3} \mathrm{O}_{4}$ and $\mathrm{Fe}_{3} \mathrm{O}_{4} / \mathrm{ZnSe}$ NP samples under zero-field-cooling (ZFC) and field-cooling (FC) conditions $(H=20 \mathrm{Oe})$. The samples were prepared in a powder form and encased in a gelatin capsule. The shape of the $M_{\mathrm{ZFC}-\mathrm{FC}}$ magnetization curves for the reference magnetite sample is that typically observed for monodispersed weakly dipole-dipole interacting systems of randomly oriented NPs, where the $M_{\mathrm{ZFC}}$ curve shows a maximum at $T_{\mathrm{M}}=32 \mathrm{~K}$ and the $M_{\mathrm{FC}}$ curve monotonically rises as $T$ decreases. For the core/shell $\mathrm{Fe}_{3} \mathrm{O}_{4} / \mathrm{ZnSe} \mathrm{NPs}$, the $M_{\mathrm{ZFC}}$ curve clearly shows a shift to higher temperatures, with a maximum at $T_{\mathrm{M}}=45 \mathrm{~K}$. Furthermore, the $M_{\mathrm{FC}}$ monotonically rises as $T$ decreases, and eventually saturates at temperatures below $41 \mathrm{~K}$. The increase in $T_{\mathrm{M}}$ from $32 \mathrm{~K}$ to $45 \mathrm{~K}$ corresponds to a slight enhancement of the magnetic anisotropy in the $\mathrm{Fe}_{3} \mathrm{O}_{4} / \mathrm{ZnSe}$ NPs. Moreover, the shift in the energy barrier $E_{\mathrm{B}}$ to higher values for the core/shell NPs should be critical in the super- paramagnetic response at room temperature. According to the Néel relaxation model, $\tau_{\mathrm{N}}=\tau_{0} \exp (\sigma)$, where $\tau_{0} \sim 10^{-10} \mathrm{~s}, \sigma$ is the ratio of anisotropy energy to thermal energy $\left(E_{\mathrm{B}} / k_{\mathrm{B}} T\right)$, and $k_{\mathrm{B}}$ is the Boltzmann constant. Therefore, the increase of $\Delta E_{\mathrm{B}} / k_{\mathrm{B}}=28 \cdot(45 \mathrm{~K}$ to $32 \mathrm{~K})=364 \mathrm{~K}$ corresponds to a threefold increase in the Néel relaxation time $\tau_{\mathrm{N}}$ at room temperature. In good agreement with the $M$ versus $T$ experiments, the increase in the coercive field from 250 Oe to 390 Oe is obtained at $T=2 \mathrm{~K}$ (figure not shown), and both samples are superparamagnetic at room temperature, i.e., without coercivity or memory effects.

The photoluminescence excitation (PLE) and PL spectra measured at room temperature for the reference $\mathrm{ZnSe}$ and core/shell $\mathrm{Fe}_{3} \mathrm{O}_{4} / \mathrm{ZnSe}$ NP samples are compared in Fig. 3(b). The core/shell $\mathrm{Fe}_{3} \mathrm{O}_{4} / \mathrm{ZnSe}$ NPs demonstrate a significant blue-shift in PL excitation/emission spectra as compared to the reference ZnSe NPs. In particular, the PL excitation/emission peaks are shifted from $377 \mathrm{~nm}$ to $325 \mathrm{~nm}$ and from 433 $\mathrm{nm}$ to $390 \mathrm{~nm}$, respectively. The observed effect is primarily due to a change of shape between spherical ZnSe NPs and nanoshell ZnSe emitters in core/shell NPs. Most dramatically, the QY dropped from $10 \%$ for the reference ZnSe NPs to $1 \%$ for the core/shell NPs. We ascribe this effect to carrier leakage at the interface between $\mathrm{Fe}_{3} \mathrm{O}_{4}$ and $\mathrm{ZnSe}$ in the core/shell NPs. Alternatively, the reduction of the QY in the ZnSe component of the core/shell NPs may be explained by the change in its geometry from spherical to a nanoshell.

In summary, we report a new colloidal synthesis method to obtain core/shell $\mathrm{Fe}_{3} \mathrm{O}_{4} / \mathrm{ZnSe} \mathrm{NPs}$. By comparing the magnetic properties of the reference $\mathrm{Fe}_{3} \mathrm{O}_{4}$ NPs and the core/shell $\mathrm{Fe}_{3} \mathrm{O}_{4} / \mathrm{ZnSe} \mathrm{NPs}$, we have shown the enhancement of the energy barrier in the core/shell composite NPs. This effect directly modifies the relaxation time, which is of fundamental interest to biomedical applications. The synthesized magnetic core/shell NPs demonstrated PL activity. However, the multicomponent NPs showed a considerable reduction in QY from $10 \%$ to $1 \%$, which could be attributed to the interface effects between the $\mathrm{Fe}_{3} \mathrm{O}_{4}$ and $\mathrm{ZnSe}$ components.

This work was supported by the National Institutes of Health under Grant No. 1R21HL092812-01A1 and by the NSF IGERT program on Integrating Nanotechnology with Cell Biology and Neuroscience, Grant No. DGE-0549500.

\footnotetext{
${ }^{1}$ N. L. Garcia-Contreras and A. Hickey, Crit. Rev. Ther. Drug Carrier Syst. 20, 317 (2003).

${ }^{2}$ N. Sanvicens and M. P. Marco, Trends Biotechnol. 26, 427 (2008).

${ }^{3}$ P. A. Jarzyna, A. Gianella, T. Skajaa, G. Knudsen, L. H. Deddens, D. P. Cormode, Z. A. Fayad, and W. J. M. Mulder, Wiley Interdisciplinary Reviews: Nanomedicine and Nanobiotechnology 2, 138 (2010).

${ }^{4}$ A. Louie, Chem. Rev. 110, 3146 (2010).

${ }^{5}$ V. I. Anisimov, I. S. Elfimov, N. Hamada, and K. Terakura, Phys. Rev. B 54, 4387 (1996).

${ }^{6}$ M. A. Hines and P. Guyot-Sionnest, J. Phys. Chem. B 102, 3655 (1998).

${ }^{7}$ P. D. Cozzoli, L. Manna, M. L. Curri, S. Kudera, C. Giannini, M. Striccoli, and A. Agostiano, Chem. Mater. 17, 1296 (2005).

${ }^{8}$ S. H. Sun and H. Zeng, J. Am. Chem. Soc. 124, 8204 (2002).

${ }^{9}$ X. Zhong, Y. Feng, Y. Zhang, Z. Gu, and L. Zou, Nanotechnology 18, 385606 (2008).

${ }^{10}$ G. Lopes, J. M. Vargas, S. K. Sharma, F. Béron, K. R. Pirota, M. Knobel, C. Rettori, and R. D. Zysler, J. Phys. Chem. C 114, 10148 (2010).

${ }^{11}$ W. Chen, S. Mørup, M. F. Hansen, T. Banert, and U. A. Peuker, J. Magn. Magn. Mater. 320, 2099 (2008).
} 